\title{
Traductores de la edición castellana
}

Enrique Alemán

Arndtstr. 28

D-1000 Berlin 61

Dr. Christof Burger

Médico

Frangenberg 19

D-5253 Lindlar

Dr. Santos Martín

Médico

Mühlenbachweg 10

D-7840 Niederweiler

Juan Luis Neumann

Traductor, BDU

Ludwig-Kirch-Platz 9

D-1000 Berlín 15
Eduardo Pardo Montero

Médico forense

E-38800 San Sebastián de la Gomera

Alfredo Muñoz Giner

Director Médico Científico

Schering España SA.

Mendez Alvaro 55

E-28045 Madrid 7

Dr. Juan Francisco Sarti

Médico

Eisenbahnstr. 14

D-8034 Germering

Stefan Schwarz

Waldstr. 57

D-1000 Berlín 21

\section{Redectores}

Regina Engst, Bibliotecaria Helmut Hildebrandt, M.A.

Karin Schick, Médica

Helmut Schwäbl †

Thomas Spitzer

Andreas Weimann, Médico

Dr. Christoph Zink, Médico 